\title{
Standounkt
}

Jugend und Nachhaltigkeit

\section{Is this what Democracy looks like?}

\begin{abstract}
Seit über einem Jahr demonstrieren Schüler/innen weltweit jeden Freitag für effektiven Klimaschutz. Doch die nötigen politischen Maßnahmen bleiben aus - welches Demokratieverständnis vermittelt solche Gehörlosigkeit jungen Menschen? Und was bleibt der Gesellschaft, um die Politik zum Handeln zu bringen? Von Therese Emilie Keil
\end{abstract}

W ir streiken, bis ihr handelt!" Unter diesem Motto demonstrieren die deutschen Fridays for Future (FFF)-Engagierten seit Dezember 2018 jeden Freitag auf den Straßen der Bundesrepublik - mit ungeahntem Erfolg. Allein am 20. September 2019 mobilisierten die Schüler/innen deutschlandweit laut Angabe von FFF 1,4 Millionen Menschen zum globalen Klimastreik. Die Protestbewegung hat dazu beigetragen, die Klimakrise innerhalb eines Jahres ganz oben auf die politische Agenda zu bringen. Leider scheint sie aber auch genau dort zu verharren. Denn an eben jenem 20. September verkündete die Große Koalition auch langerwartete Klimaschutzmaßnahmen, die von den Aktivist/innen allenfalls als „Klimapaketchen“ belächelt werden. Auf die Abschwächung des sowieso schon unzureichenden Klimapaketes folgte ein Kohleausstiegsgesetz, welches eine schnelle Energiewende und damit die Einhaltung des Pariser 1,5 Grad-Zieles undenkbar machte. Passend dazu titelte ein Banner der Demonstrant/innen bei der Bekanntmachung der Empfehlungen der Kohlekommission: „Wir haben nichts erwartet, und sind trotzdem enttäuscht.“

\section{Von Politikverdrossen- heit zu professionellem Engagement}

Die FFF-Bewegung ist historisch neu, noch nie organisierten Unter-18-Jährige so professionell politisches Engagement. Deshalb spricht man gerne davon, dass mit den Klimastreiks eine ganze Generation politisiert wurde. Das Handlungsdefizit der Bundesregierung birgt wiederum leider das Potenzial, eine ganze Generation in die Politikverdrossenheit zu treiben. Denn nichts bringt die mangelhaften Partizipationsmöglichkeiten unserer parlamentarischen Demokratie besser zum Ausdruck, als die Tatsache, dass seit einem Jahr Millionen junge Menschen für ihre Zukunft demonstrieren, während die Regierung das Kohlekraftwerk Datteln IV ans Netz gehen lässt. Der verfehlte Kohleausstieg ist ein Beispiel für eine Politik, die Konzerninteressen über den Erhalt unseres Planeten stellt.

\section{Radikale Protestformen als Ausweg?}

Da ist es wenig verwunderlich, dass inzwischen auch die FFF-Aktivist/innen zu radikaleren Protestformen aufrufen. Bis vor wenigen Monaten wurde medial gerne ein Gegensatz-Narrativ zwischen den „brav demonstrierenden Schüler/innen " und den "gewaltbereiten Klimaaktivist/innen"von Ende Gelände, Extinction Rebellion \& Co kreiert. Doch spätestens seit im November 2019 FFF und Ende Gelände unter dem Motto „Freitags demonstrieren, samstags blockieren“ gemeinsam zu Aktionen zivilen Ungehorsams im Lausitzer Braunkohlerevier aufriefen, ist klar: die deutsche Klimaschutzbewegung ist unzertrennlich und wenn die Regierung versagt, nehmen sie den Klimaschutz eben selbst in die Hand.

\section{Generationenkonflikt in der deutschen Gesellschaft}

FFF steht jedoch nicht repräsentativ für die deutsche Gesellschaft, vielmehr zeugt die öffentliche Debatte um sie von einem Generationenkonflikt, der sich durch die gesamte Umweltschutzdiskussion zieht. So beleidigen manche Politiker/innen und einzelne Medien die Aktivist/innen als „opportunistische Schulschwänzer/innen“. Gleichzeitig wirft die Jugend den älteren Generationen (den „Babyboomern“) vor, ihren Wohlstand auf Kosten zukünftiger Generationen aufgebaut $\mathrm{zu}$ haben. Diese wiederum fragen polemisch nach seltenen Erden in Smartphones und kritisieren damit implizit das Mediennutzungsverhalten junger Menschen - und überhaupt, wie oft fliegt Luisa Neubauer eigentlich in den Urlaub? Doch Debatten um den Individualkonsum sind zwar grundsätzlich wichtig, lenken aber viel zu oft vom eigentlichen Problem ab. Denn die so erreichten $\mathrm{CO}_{2}$-Einsparungen können immer nur ein Bruchteil dessen sein, was Kohlekonzerne und Autoindustrie jährlich für ihren Profit verheizen. Die Ausmaße der Klimakrise sind bekannt und die Durchsetzung der nötigen Maßnahmen eine Frage des politischen Willens.

Die Klimagerechtigkeitsbewegung hat verstanden, dass ihr kollektives Anliegen wichtiger ist, als der Unterschied in ihren Aktionsformen, und mobilisiert seitdem Seite an Seite. Davon könnte die Gesellschaft lernen, Generationskonflikte ebenso dem gemeinsamen Ziel unterzuordnen, um vereint eine mutige Politik einzufordern - schließlich geht es um nicht weniger als den Erhalt unseres Lebensraumes.

AUTORIN + KONTAKT

Therese Emilie Keil studiert Liberal Arts and Science an der Universität Maastricht und war Mitglied des Jugendbeirats des IÖW-Projekts Zukunft? Jugend fragen! Sie engagiert sich als Klimaaktivistin.

E-Mail: therese.keil@gmx.de, Tel.: +4915780657942
ÖkologischesWirtschaften 2.2020 (35) | DOI 10.14512/OEW350210

C 2020 T. E. Keil; licensee IÖW and oekom verlag. This is an article distributed under the terms of the Creative Commons Attribution Non-Commercial No Derivates License (http://creativecommons.org/licenses/by-nc-nd/4.o/deed.de), which permits copying and redistributing the material in any medium or format, provided the original work is properly cited, it is not used for commercial purposes and it is not remixed, transformed or built upon. The access to the digital version of this article is reserved to subscribers of ÖkologischesWirtschaften until two years after the date of publication; after two years it is available to all readers. 